'Departamento de Nutrición y Dietética, Facultad de Farmacia, Universidad de Concepción. Concepción, Chile. ${ }^{2}$ Centro de Vida Saludable, Universidad de Concepción. Concepción, Chile. ${ }^{3}$ Instituto de Farmacia, Facultad de Ciencias, Universidad Austral de Chile. Valdivia, Chile.

${ }^{4}$ Departamento de Psicología, Facultad de Ciencias Sociales, Universidad de Concepción. Concepción, Chile.

${ }^{5}$ Centro de Investigación en Educación y Desarrollo (CIEDE-UCSC), Departamento de Salud Pública, Facultad de Medicina, Universidad Católica de la Santísima Concepción. Concepción, Chile.

${ }^{6}$ Departamento de Bioquímica Clínica e Inmunología, Facultad de Farmacia. Universidad de Concepción. Concepción Chile.

${ }^{7}$ Centro de Investigación en Fisiología de Ejercicio (CIFE), Universidad Mayor. Santiago, Chile.

${ }^{8}$ Laboratorio de Rendimiento Humano, Grupo de Estudio en Educación, Actividad

Física y Salud (GEEAFyS), Universidad Católica del Maule. Talca, Chile.

Institute of Health and Wellbeing, University of Glasgow. Glasgow, United Kingdom. ${ }^{10} \mathrm{BHF}$ Glasgow Cardiovascular Research

Centre, Institute of Cardiovascular and Medical Sciences, University of Glasgow. Glasgow, United Kingdom.

'Facultad de Medicina, Universidad Diego Portales. Santiago, Chile. ${ }^{a}$ Nutricionista. bBioquímico. 'Psicóloga. dProfesor de Educación física. ${ }^{\mathrm{e} M S C}$ ${ }^{\mathrm{f}} \mathrm{PhD}$.

*KRA, AML y MM contribuyeron de igual forma a este manuscrito y son considerados primer autor compartido.

Trabajo no recibió financiamiento Los autores declaran no tener conflictos de interés.

Recibido el 1 de julio de 2020, aceptado el 4 de marzo de 2021

Correspondencia a: Fanny Petermann-Rocha BHF Glasgow Cardiovascular Research Centre Institute of Cardiovascular and Medical Sciences. College of Medical, Veterinary and Life Sciences. University of Glasgow.

Glasgow, G12 8TA, United Kingdom f.petermann-rocha.1@research.gla.ac.uk

\section{Caracterización del consumo de legumbres en población chilena: resultados de la Encuesta Nacional de Salud 2016-2017}

\author{
KARINA RAMÍREZ-ALARCÓN ${ }^{1, \mathrm{a}, \mathrm{e}, *,}$ \\ ANA MARÍA LABRAÑA ${ }^{1, \mathrm{a}, \mathrm{e}, *}$, MIQUEL MARTORELL ${ }^{1,2, \mathrm{~b}, \mathrm{f}, *}$, \\ MARÍA ADELA MARTÍNEZ-SANGUINETTI ${ }^{3, b, e}$, \\ GABRIELA NAZAR ${ }^{2,4, \mathrm{c}, \mathrm{f}}$, CLAUDIA TRONCOSO-PANTOJA ${ }^{5, \mathrm{a}, \mathrm{e}}$, \\ NATALIA ULLOA ${ }^{2,6, b}$, CARLOS CELIS-MORALES ${ }^{7,8,9,10, \mathrm{~d}, \mathrm{f}}$, \\ FANNY PETERMANN-ROCHA ${ }^{10,11, a, e}$ \\ (en representación del Consorcio de Investigación ELHOC)
}

\section{Legume consumption in chileans: findings from the 2016-2017 national health survey}

Background: Legumes are low cost and high-quality nutritional foods. In Chile, a twice per week legume consumption is recommended to promote health and prevent disease. Aim: To characterize the consumption of legumes according to sociodemographic and anthropometric variables in the Chilean adult population. Material and Method: Analysis of data from 5,473 participants of the 2016-2017 National Health Survey. The compliance with legume consumption was studied in population groups, according to sociodemographic and anthropometric characteristics, through logistic regression analyses. Results: Only 24\% of all participants (51.4\% of women) fulfilled legume intake recommendations. After adjusting for sociodemographic variables, the participants who were less likely to comply with the recommendation were widowers (Odds Ratio (OR): 0.58 [95\% confidence intervals (CI): (0.40; 0.85]). On the contrary, people between 70-80 years (OR: 1.78 [95\% CI: 1.11; 2.88]), those who resided in rural areas (OR: 1.62 [95\% CI: 1.25; 2.10]) and those who resided in the Maule region (OR: 2.11 [95\% CI: 1.37, 3.25]) had a higher likelihood of compliance. Conclusions: One out of four Chileans complied with the recommendations of legume consumption. Even though the results differed when stratified by sex, it is highlighted that living in rural areas increased the probabilities of an adequate legume consumption.

(Rev Med Chile 2021; 149: 698-707)

Key words: Fabaceae; Health Care Surveys; Nutrition Policy. 
L as leguminosas pertenecen a la familia vegetal Leguminosae y son el tercer grupo de plantas más numeroso del planeta ${ }^{1}$. Se consideran legumbres a las semillas secas comestibles que se producen dentro de una vaina. Son alimentos de bajo costo y alta calidad nutricional y pueden contribuir a mejorar la densidad de nutrientes de la dieta debido a su alto contenido de proteínas (20\%-30\%), aminoácidos esenciales, tales como lisina y treonina, alto aporte de carbohidratos complejos (50\%-65\%), bajo aporte de lípidos (cerca de 1\%) y un bajo índice glicémico ${ }^{3}$. Son fuente de vitaminas del complejo B (niacina, ácido fólico, tiamina y riboflavina) y minerales (hierro no hemínico, zinc, calcio, magnesio, fósforo y cobre $)^{3-9}$. Sin embargo, pueden tener niveles bajos de aminoácidos esenciales azufrados (metionina y cisteína), por lo que se recomienda consumirlas mezcladas con cereales ricos en estos, obteniéndose una mezcla de proteínas de un alto valor nutricional ${ }^{10}$. Además, contienen sustancias bioactivas que no se consideran nutrientes, pero ejercen efectos metabólicos favorables en el organismo, tales como fibra dietética, almidones resistentes y fitoquímicos bioactivos, principalmente polifenoles y fitoesteroles ${ }^{11}$. La ingesta de legumbres podría controlar la glicemia en personas diabéticas, reducir el riesgo de eventos cardiovasculares, cáncer, presión arterial, niveles de colesterol total, lipoproteínas de baja densidad y triglicéridos ${ }^{4,12,13}$. De los 11 millones de muertes a nivel mundial atribuibles a una alimentación no saludable, se estima que aproximadamente $5 \%$ de los decesos producidos por enfermedades cardiovasculares son atribuibles a un consumo sub-óptimo de legumbres, lo que podría prevenirse con una ingesta de 50-70 g/día ${ }^{10,14-16}$.

En Chile, las recomendaciones de ingesta de legumbres son establecidas por el Ministerio de Salud (MINSAL), elaboradas en base a las directrices de la Organización de las Naciones Unidas para la Alimentación y la Agricultura, en la forma de Guías Alimentarias Basadas en Alimentos ${ }^{17}$. Éstas recomiendan el consumo de legumbres 2 veces por semana lo que equivale aproximadamente a 20 g/día (calculado a partir de una porción de 70 g), sin mezclarlas con cecinas. Se destacan por ser accesibles, nutritivas y económicas, posicionándose como un alimento estratégico en la seguridad alimentaria nutricional de la población, en casos de emergencia o catástrofes como la pandemia por COVID-19 ${ }^{1,17,18}$. Sin embargo, en Chile, según la Encuesta Nacional de Consumo Alimentario (ENCA 2010), solo 25,1\% de la población cumple con la recomendación, con una ingesta proporcional de $17,7 \mathrm{~g} /$ día por persona ${ }^{18}$. Por lo anterior, el objetivo de este trabajo fue caracterizar el consumo de legumbres, según variables sociodemográficas y antropométricas en población adulta chilena.

\section{Metodología}

\section{Diseño del estudio}

Estudio observacional de corte transversal que incluyó a 5.473 personas $\geq 15$ años, que participaron en la Encuesta Nacional de Salud realizada entre los años 2016 y 2017 (ENS 2016-2017) y que contaban con información sobre el consumo de legumbres y variables sociodemográficas y antropométricas. El número de participantes equivale a una muestra expandida de 14.431 .358 chilenos de ambos sexos.

La ENS 2016-2017 implementó un muestreo aleatorio estratificado para reclutar participantes de áreas rurales y urbanas con una distribución equitativa aproximada. Fue financiada por el MINSAL y aprobada por el Comité Ético Científico de la Facultad de Medicina de la Pontificia Universidad Católica de Chile. Todos los participantes dieron su consentimiento informado por escrito antes del comienzo del estudio ${ }^{19,20}$. Los datos utilizados en este trabajo fueron solicitados al MINSAL, según el protocolo establecido.

\section{Consumo de legumbres}

La ingesta diaria fue evaluada a través de la pregunta: ¿Con quéfrecuencia consume usted algún tipo de legumbres como porotos, lentejas, arvejas o garbanzos?, para cumplir con la recomendación de ingesta, el individuo debía declarar consumir al menos 2 veces/semana algún tipo de legumbres ${ }^{21}$. Para evaluar el cumplimiento de la recomendación acorde a segmentos de población, estos fueron divididos en grupos según características sociodemográficas y antropométricas.

\section{Variables sociodemográficas}

Fueron recolectadas utilizando cuestionarios previamente validados por la ENS 2016-2017 ${ }^{19}$. Las variables fueron: edad, sexo, área geográfica, 
nivel educacional, nivel educacional jefe de hogar y nivel socioeconómico (NSE), clasificado según la Sociedad de Investigación de Mercados y Opinión $(\text { ESOMAR })^{22}$. Otras variables consideradas fueron ocupación, regiones de Chile y estado civil.

\section{Variables antropométricas}

La medición del peso y talla corporal fueron recolectados mediante métodos estandarizados, tal como ha sido reportado anteriormente ${ }^{19,23}$. El estado nutricional se clasificó según índice masa corporal (IMC) en base a las recomendaciones de la OMS para adultos ${ }^{24}$. Para los adultos mayores se utilizaron los puntos de corte de IMC establecidos por el MINSAL ${ }^{25}$. La obesidad central fue definida por circunferencia de cintura (CC) como $\geq 88$ y $\geq 102 \mathrm{~cm}$ en mujeres y hombres, respectivamente ${ }^{20,23}$.

\section{Análisis estadístico}

Se utilizó el programa STATA MP v16 (StataCorp; College Station, TX) y el comando de "svyset" para muestras complejas. Los resultados fueron estimados utilizando muestras expandidas acorde a factores sugeridos por ENS 2016-2017. Los análisis fueron ponderados por el diseño de la encuesta. Las características descriptivas de la cohorte se presentan como medias o proporción con sus intervalos de confianza de 95\% (IC de 95\%) para las variables continuas y categóricas.

Para investigar las asociaciones entre el consumo de legumbres con factores sociodemográficos y antropométricos, se realizaron análisis de regresión logística con los resultados presentados como Odds Ratios (OR), con sus respectivos 95\% IC. Para determinar la probabilidad de cumplir la recomendación del consumo de legumbres, se utilizó como grupo de referencia a aquellas personas que reportaron consumir $\geq 2$ veces a la semana legumbres. Por lo que un OR $>1$ sugiere una mayor probabilidad de cumplir, mientras que un OR $<1$ indica una menor probabilidad de cumplir con la recomendación.

Todos los análisis estadísticos se ajustaron de forma incremental mediante factores de confusión, incluidos en 2 modelos estadísticos: Modelo 1- no ajustado; Modelo 2 - ajustado por variables sociodemográficas (edad, sexo y nivel educacional); para evitar colinealidad, el NSE no fue ajustado por nivel educacional en ninguno de los modelos. Finalmente, para investigar si las asociaciones diferían por subgrupos, se realizó un análisis estratificado por sexo utilizando el modelo 2 (no fue ajustado por sexo). El nivel de significancia fue definido como $p<0,05$.

\section{Resultados}

En la Tabla 1 se muestran las características de los participantes según el cumplimiento de la recomendación del consumo de legumbres. Solo $24 \%$ de los participantes cumplían con esta recomendación, 51,4\% eran mujeres. La edad promedio fue de 46,3 años, $75,7 \mathrm{~kg}$ de peso corporal, talla de 1,62 m, una CC de 93,9 cm y un IMC de $28,7 \mathrm{~kg} / \mathrm{m}^{2}$. En relación con el estado nutricional, quienes cumplían, presentaban una mayor probabilidad de tener sobrepeso $(40,2 \%)$ y obesidad central $(46,7 \%)$.

En la Tabla 2 se presenta la probabilidad de cumplir la recomendación de consumo de legumbres acorde a características sociodemográficas y antropométricas de los participantes de la ENS 2016-2017, según 2 modelos estadísticos. En el modelo 1 , se destaca que presentaron menor probabilidad de cumplir con la recomendación, los participantes con nivel educacional superior (OR: 0,60 [95\% IC: 0,44; 0,83]), respecto de los con nivel educacional básico; y los solteros/as (OR: 0,64 [95\% IC: 0,50; 0,84), respecto de los casados/as. Al contrario, presentaron una mayor probabilidad de cumplir la recomendación, las personas > 80 años (OR: 1,95 [95\% IC: 1,00; $3,80]$ ), respecto de los < 20 años (Figura 1); los jubilados/as (OR: 1,48 [95\% IC: 1,10; 1,99]), respecto de personas laboralmente activas; los que residen en área rural (OR: 1,73 [95\% IC: 1,35; $2,22]$ ), respecto de los que viven en área urbana; los habitantes de las regiones de Arica y Parinacota, Tarapacá, Antofagasta, Atacama, O’Higgins, Maule, Biobío, Araucanía y Los Ríos, presentaron una mayor probabilidad de cumplimiento $y$, de estas regiones, las que mostraron mayor cumplimiento fueron la región del Maule (OR: 2,12 [95\% IC: 1,38; 3,25]) y Biobío (OR: 2,09 [95\% IC: 1,49; $2,92]$ ), respecto de la región Metropolitana.

Cuando el modelo 2 fue ajustado por las variables de confusión, las asociaciones que tenían menor cumplimiento desaparecieron; sin embargo, presentaron una menor probabilidad de cumplimiento los viudos/as (42\% menor de 
Tabla 1. Características de los participantes ENS 2016-2017, según cumplimiento del consumo de legumbres

\begin{tabular}{|c|c|c|}
\hline Características & $\begin{array}{c}\text { No cumplen } \\
\text { (< } 2 \text { veces/semana) }\end{array}$ & $\begin{array}{c}\text { Cumplen } \\
\text { (> } 2 \text { veces/semana) }\end{array}$ \\
\hline Participantes encuestados & $\mathrm{n}=4.159(76 \%)$ & $n=1.313(24 \%)$ \\
\hline Muestra expandida & $n=10.922 .910(75,7 \%)$ & $n=3.508 .447(24,3 \%)$ \\
\hline Cumplimiento de recomendación de porciones de legumbres (\%) & $76(74 ; 77)$ & $24(22 ; 26)$ \\
\hline \multicolumn{3}{|l|}{ Sexo } \\
\hline Hombre (\%) & $49,2(46,5 ; 52,0)$ & $48,6(44,1 ; 53,2)$ \\
\hline Mujer (\%) & $50,8(48,0 ; 53,5)$ & $51,4(46,8 ; 55,9)$ \\
\hline Edad (años) & $42,1(41,2 ; 43,0)$ & $46,3(44,7 ; 47,9)$ \\
\hline Peso corporal (kg) & $75,5(74,6 ; 76,4)$ & $75,7(74,4 ; 76,9)$ \\
\hline Talla (m) & $1,6(1,6 ; 1,6)$ & $1,6(1,6 ; 1,6)$ \\
\hline IMC $\left(\mathrm{kg} / \mathrm{m}^{2}\right)$ & $28,5(28,2 ; 28,8)$ & $28,7(28,2 ; 29,1)$ \\
\hline \multicolumn{3}{|l|}{ Estado nutricional } \\
\hline Bajo peso (\%) & $2,9(2,2 ; 3,8)$ & $3,3(2,1 ; 5,0)$ \\
\hline Normopeso (\%) & $27,8(24,5 ; 30,4)$ & $25,9(22,2 ; 30,1)$ \\
\hline Sobrepeso (\%) & $37,6(34,9 ; 40,3)$ & $40,2(35,7 ; 44,8)$ \\
\hline Obeso (\%) & $31,7(29,2 ; 34,3)$ & $30,6(26,6 ; 34,8)$ \\
\hline Circunferencia cintura $(\mathrm{cm})$ & $93,1(92,3 ; 93,8)$ & $93,9(92,7 ; 95,1)$ \\
\hline \multicolumn{3}{|l|}{ Obesidad Central } \\
\hline Sin obesidad central (\%) & $56,9(54,2 ; 59,6)$ & $53,3(48,9 ; 57,9)$ \\
\hline Con obesidad central (\%) & $43,1(40,4 ; 45,8)$ & $46,7(42,1 ; 51,1)$ \\
\hline \multicolumn{3}{|l|}{ Área geográfica } \\
\hline Urbana (\%) & $90,5(89,2 ; 91,5)$ & $84,6(81,7 ; 87,1)$ \\
\hline Rural (\%) & $9,5(8,5 ; 10,8)$ & $15,5(12,9 ; 18,4)$ \\
\hline Escolaridad (años) & $11,1(10,9 ; 11,3)$ & $10,5(10,2 ; 10,9)$ \\
\hline \multicolumn{3}{|l|}{ Nivel educacional } \\
\hline Básico ( $\leq 8$ años) (\%) & $15,6(13,9 ; 17,4)$ & $19,3(16,2 ; 22,7)$ \\
\hline Medio (9-12 años) (\%) & $55,0(52,2 ; 57,8)$ & $58,8(54,3 ; 63,2)$ \\
\hline Superior ( $\geq 12$ años) (\%) & $29,4(26,8 ; 32,2)$ & $21,9(18,2 ; 26,2)$ \\
\hline \multicolumn{3}{|l|}{ Nivel socio-económico } \\
\hline Bajo (\%) & $25,7(23,5 ; 28,1)$ & $27,5(23,6 ; 31,7)$ \\
\hline Medio (\%) & $31,1(28,4 ; 33,9)$ & $36,8(32,0 ; 41,9)$ \\
\hline Alto (\%) & $43,2(40,2 ; 46,3)$ & $35,7(30,9 ; 40,7)$ \\
\hline
\end{tabular}

IMC: índice masa corporal. Datos presentados como media y su 95\% IC para variables continuas y como porcentaje (\%) y su 95\% IC para variables categóricas. Todos los valores fueron corregidos por el factor de expansión sugerido por la ENS $2016-$ 2017.

probabilidad) en relación con los casados. Una mayor probabilidad de cumplimiento se mantuvo en personas que vivían en áreas rurales, entre los grupos etarios de 60-70 años y el de 70-80 años (64\% y $78 \%$ de cumplimiento, respectivamente) (Figura 1), así como los habitantes de las regiones de Arica y Parinacota, Tarapacá, Antofagasta, Atacama, O’Higgins, Maule, Biobío, y Los Ríos (entre 60-106\% de mayor cumplimiento).

Cuando los análisis fueron estratificados por sexo (Figura 2), las mujeres entre 30 a 80 años presentaron una mayor probabilidad de cumpli- 
Tabla 2. Probabilidad de cumplir la recomendación de ingesta de legumbres acorde a características sociodemográficas y antropométricas de la ENS 2016-2017, según 2 modelos estadísticos

\begin{tabular}{|c|c|c|c|c|c|c|}
\hline \multirow{2}{*}{$\begin{array}{l}\text { Sexo* } \\
\text { Masculino (Ref.) } \\
\text { Femenino }\end{array}$} & \multicolumn{2}{|r|}{$\begin{array}{l}\text { Modelo } 1 \\
5 \% \text { IC] }\end{array}$} & $\mathbf{p}$ & \multicolumn{2}{|r|}{$\begin{array}{l}\text { Modelo } 2 \text { * } \\
5 \% \text { IC] }\end{array}$} & \multirow[b]{2}{*}{0,937} \\
\hline & $\begin{array}{l}1,00 \\
1,02\end{array}$ & {$[0,83 ; 1,27]$} & 0,822 & $\begin{array}{l}1,00 \\
0,99\end{array}$ & {$[0,79 ; 1,23]$} & \\
\hline $\begin{array}{l}\text { Estado Nutricional } \\
\text { Normal (Ref.) } \\
\text { Bajo Peso } \\
\text { Sobrepeso } \\
\text { Obeso }\end{array}$ & $\begin{array}{l}1,00 \\
1,20 \\
1,14 \\
1,04\end{array}$ & $\begin{array}{l}{[0,69 ; 2,09]} \\
{[0,87 ; 1,50]} \\
{[0,79 ; 1,36]}\end{array}$ & $\begin{array}{l}0,515 \\
0,325 \\
0,799\end{array}$ & $\begin{array}{l}1,00 \\
0,96 \\
1,11 \\
0,98\end{array}$ & $\begin{array}{l}{[0,54 ; 1,71]} \\
{[0,85 ; 1,46]} \\
{[0,75 ; 1,28]}\end{array}$ & $\begin{array}{l}0,886 \\
0,440 \\
0,867\end{array}$ \\
\hline $\begin{array}{l}\text { Obesidad Central } \\
\text { No (Ref.) } \\
\text { Si }\end{array}$ & $\begin{array}{l}1,00 \\
1,15\end{array}$ & {$[0,93 ; 1,42]$} & 0,191 & $\begin{array}{l}1,00 \\
1,03\end{array}$ & {$[0,82 ; 1,29]$} & 0,814 \\
\hline $\begin{array}{l}\text { Estado civil } \\
\text { Casado (Ref.) } \\
\text { Convive (con o sin acuerdo) } \\
\text { Divorciado/separado/anulado } \\
\text { Viudo/a } \\
\text { Soltero/a }\end{array}$ & $\begin{array}{l}1,00 \\
0,94 \\
0,88 \\
0,76 \\
0,64\end{array}$ & $\begin{array}{l}{[0,68 ; 1,31]} \\
{[0,58 ; 1,35]} \\
{[0,54 ; 1,08]} \\
{[0,50 ; 0,84]}\end{array}$ & $\begin{array}{l}0,728 \\
0,562 \\
0,133 \\
0,001\end{array}$ & $\begin{array}{l}1,00 \\
1,11 \\
0,87 \\
0,58 \\
0,84\end{array}$ & $\begin{array}{l}{[0,79 ; 1,56]} \\
{[0,57 ; 1,32]} \\
{[0,40 ; 0,85]} \\
{[0,63 ; 1,13]}\end{array}$ & $\begin{array}{l}0,532 \\
0,517 \\
0,005 \\
0,258\end{array}$ \\
\hline $\begin{array}{l}\text { Nivel educacional* } \\
\text { Básico (<9 años) (Ref.) } \\
\text { Medio ( } 9-12 \text { años) } \\
\text { Superior ( }>12 \text { años) }\end{array}$ & $\begin{array}{l}1,00 \\
0,86 \\
0,60\end{array}$ & $\begin{array}{l}{[0,67 ; 1,11]} \\
{[0,44 ; 0,83]}\end{array}$ & $\begin{array}{l}0,267 \\
0,002\end{array}$ & $\begin{array}{l}1,00 \\
1,11 \\
0,80\end{array}$ & $\begin{array}{l}{[0,84 ; 1,47]} \\
{[0,56 ; 1,13]}\end{array}$ & $\begin{array}{l}0,460 \\
0,211\end{array}$ \\
\hline $\begin{array}{l}\text { Nivel educacional jefe de hogar } \\
\text { Básico (<9 años) (Ref.) } \\
\text { Medio ( } 9-12 \text { años) } \\
\text { Superior ( }>12 \text { años) }\end{array}$ & $\begin{array}{l}1,00 \\
0,83 \\
0,69\end{array}$ & $\begin{array}{l}{[0,55 ; 1,24]} \\
{[0,41 ; 1,16]}\end{array}$ & $\begin{array}{l}0,357 \\
0,161\end{array}$ & $\begin{array}{l}1,00 \\
0,87 \\
0,72\end{array}$ & $\begin{array}{l}{[0,57 ; 1,33]} \\
{[0,42 ; 1,24]}\end{array}$ & $\begin{array}{l}0,528 \\
0,241\end{array}$ \\
\hline $\begin{array}{l}\text { Nivel socioeconómico } \\
\text { Bajo (Ref.) } \\
\text { Medio } \\
\text { Alto }\end{array}$ & $\begin{array}{l}1,00 \\
1,11 \\
0,77\end{array}$ & $\begin{array}{l}{[0,84 ; 1,46]} \\
{[0,59 ; 1,01]}\end{array}$ & $\begin{array}{l}0,470 \\
0,068\end{array}$ & $\begin{array}{l}1,00 \\
1,16 \\
0,85\end{array}$ & $\begin{array}{l}{[0,88 ; 1,53]} \\
{[0,64 ; 1,14]}\end{array}$ & $\begin{array}{l}0,289 \\
0,284\end{array}$ \\
\hline $\begin{array}{l}\text { Ocupación } \\
\text { Trabajador (Ref.) } \\
\text { Dueña de casa } \\
\text { Estudiante } \\
\text { Jubilado/a }\end{array}$ & $\begin{array}{l}1,00 \\
1,22 \\
0,84 \\
1,48\end{array}$ & $\begin{array}{l}{[0,92 ; 1,61]} \\
{[0,58 ; 1,22]} \\
{[1,10 ; 1,99]}\end{array}$ & $\begin{array}{l}0,174 \\
0,359 \\
0,008\end{array}$ & $\begin{array}{l}1,00 \\
1,16 \\
1,20 \\
0,90\end{array}$ & $\begin{array}{l}{[0,82 ; 1,64]} \\
{[0,79 ; 1,83]} \\
{[0,62 ; 1,33]}\end{array}$ & $\begin{array}{l}0,392 \\
0,396 \\
0,624\end{array}$ \\
\hline $\begin{array}{l}\text { Regiones } \\
\text { Metropolitana (Ref.) } \\
\text { Arica y Parinacota } \\
\text { Tarapacá } \\
\text { Antofagasta } \\
\text { Atacama } \\
\text { Coquimbo } \\
\text { Valparaiso } \\
\text { O’Higgins } \\
\text { Maule } \\
\text { Biobío } \\
\text { Araucania } \\
\text { Los Ríos } \\
\text { Los Lagos } \\
\text { Aysén } \\
\text { Magallanes y la Antártica }\end{array}$ & $\begin{array}{l}1,00 \\
1,59 \\
1,60 \\
1,94 \\
1,84 \\
1,51 \\
1,40 \\
1,77 \\
2,12 \\
2,09 \\
1,51 \\
1,70 \\
1,52 \\
1,29 \\
0,82\end{array}$ & $\begin{array}{l}{[1,02 ; 2,46]} \\
{[1,01 ; 2,52]} \\
{[1,27 ; 2,98]} \\
{[1,06 ; 3,18]} \\
{[0,96 ; 2,37]} \\
{[0,96 ; 1,96]} \\
{[1,03 ; 3,01]} \\
{[1,38 ; 3,26]} \\
{[1,49 ; 2,92]} \\
{[1,00 ; 2,28]} \\
{[1,06 ; 2,70]} \\
{[0,96 ; 2,41]} \\
{[0,85 ; 1,97]} \\
{[0,48 ; 1,43]}\end{array}$ & $\begin{array}{c}0,039 \\
0,044 \\
0,002 \\
0,028 \\
0,070 \\
0,079 \\
0,038 \\
0,001 \\
<0,001 \\
0,049 \\
0,026 \\
0,075 \\
0,235 \\
0,491\end{array}$ & $\begin{array}{l}1,00 \\
1,60 \\
1,66 \\
1,99 \\
1,86 \\
1,50 \\
1,36 \\
1,74 \\
2,11 \\
2,06 \\
1,48 \\
1,65 \\
1,49 \\
1,29 \\
0,82\end{array}$ & $\begin{array}{l}{[1,03 ; 2,49]} \\
{[1,05 ; 2,63]} \\
{[1,30 ; 3,05]} \\
{[1,06 ; 3,25]} \\
{[0,96 ; 2,35]} \\
{[0,95 ; 1,94]} \\
{[1,03 ; 2,92]} \\
{[1,37 ; 3,25]} \\
{[1,47 ; 2,87]} \\
{[0,99 ; 2,21]} \\
{[1,03 ; 2,65]} \\
{[0,93 ; 2,39]} \\
{[0,84 ; 1,96]} \\
{[0,47 ; 1,43]}\end{array}$ & $\begin{array}{c}0,036 \\
0,031 \\
0,001 \\
0,029 \\
0,072 \\
0,091 \\
0,036 \\
0,001 \\
<0,001 \\
0,056 \\
0,037 \\
0,098 \\
0,242 \\
0,489\end{array}$ \\
\hline $\begin{array}{l}\text { Área Geográfica } \\
\text { Urbana (Ref.) } \\
\text { Rural }\end{array}$ & $\begin{array}{l}1,00 \\
1,73\end{array}$ & {$[1,35 ; 2,22]$} & $<0,001$ & $\begin{array}{l}1,00 \\
1,62\end{array}$ & {$[1,25 ; 2,10]$} & $<0,001$ \\
\hline
\end{tabular}

Referencia (Ref.). Cumplimiento recomendación ingesta de legumbres (2 veces/semana). Datos representados como Odds Ratio (OR) y su respectivo 95\% IC. Modelo 1 no ajustado, Modelo 2 ajustado por variables sociodemográficas (edad, sexo y nivel educacional). *En Modelo 2 las variables edad, sexo y nivel educacional no están ajustados por sí mismas. 


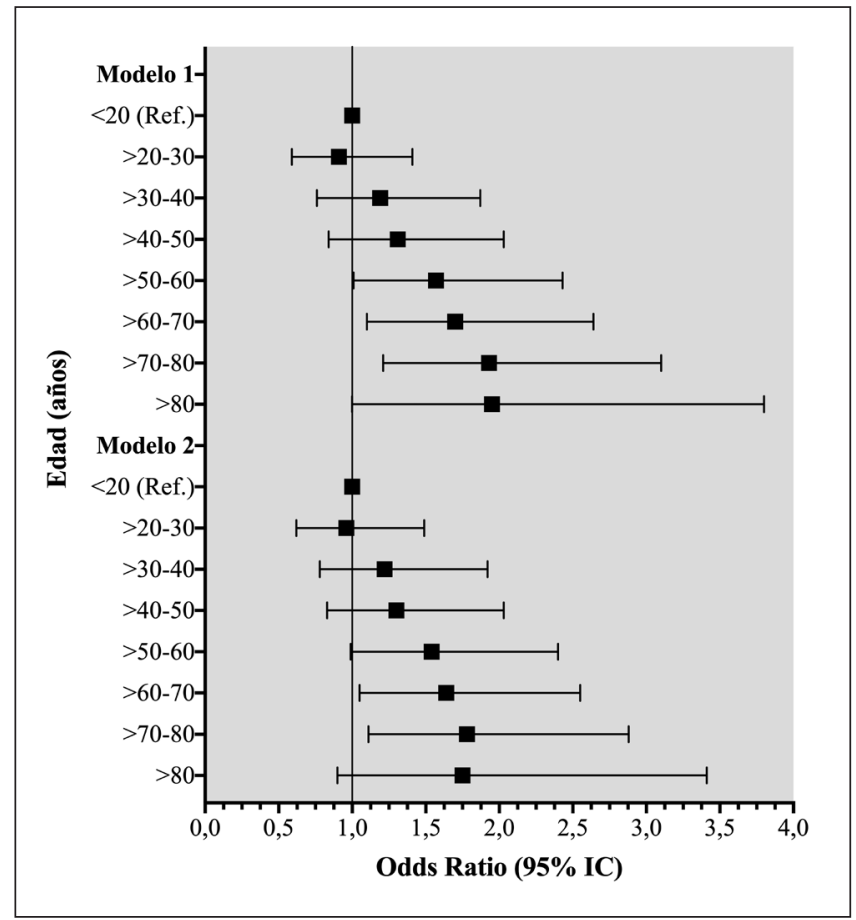

Figura 1. Cumplimiento recomendación ingesta de legumbres (2 veces/semana) según grupos de edad. Datos representados como Odds Ratio (OR) y su respectivo 95\% IC. Modelo 1 no ajustado, modelo 2 ajustado por variables sociodemográficas (edad, sexo y educación). En el modelo 2 la edad, sexo, ni educación están ajustadas por sí misma. (Ref.): Referencia. miento y las entre 70-80 años mostraron una mayor probabilidad (OR: 2,61 [95\% IC: 1,34; 5,10]). En el caso de las regiones, los hombres del Maule y Biobío reportaron un mayor probabilidad de cumplimiento (OR:3,59 [95\% IC: 1,$8 ; 7,16]$ y OR $2,17$ [95\% IC: 1,$27 ; 3,72]$, respectivamente) y en el caso de las mujeres, solo aquellas pertenecientes a la región del Biobío (OR: 1,96 [95\% IC: 1,29; $2,97]$ ). Las personas que residían en zona rural reportaron una mayor probabilidad de cumplimiento en ambos sexos en comparación a zonas urbanas $\left(\mathrm{OR}_{\text {hombres }}: 1,74\right.$ [95\% IC: 1,$\left.11 ; 2,71\right] \mathrm{y}$ OR $_{\text {muieres }}: 1,49$ [95\% IC: 1,$\left.\left.10 ; 2,02\right]\right)$. Finalmente, hombres con un nivel educacional medio o alto presentaron una menor probabilidad de cumplimiento (Figura 2).

\section{Discusión}

El consumo de legumbres 2 veces por semana es beneficioso para la nutrición y salud de las personas y es una estrategia para promover una alimentación saludable, y mantener un buen estado de salud ${ }^{3}$. En este estudio, solo $24 \%$ de los participantes cumplían con las recomendaciones de consumo de legumbres 2 veces/semana, lo que equivale aproximadamente a $20 \mathrm{~g} /$ día, cumpliendo las mujeres en mayor proporción. Resultados parcialmente similares reportó la ENCA 2010, donde el consumo de legumbres fue de $25,1 \%$, con un promedio de consumo de 17,7 g/día; sin embargo, los hombres reportaron un mayor consumo, con 21,4 g/día. A pesar de que transcurrieron 7 años desde su aplicación, la población chilena no ha aumentado la ingesta de este grupo de alimentos $^{8,18}$. Otro estudio publicado en Chile el año 2019 , reportó que solo $23 \%$ de sus participantes cumplía con la recomendación del consumo de legumbres, siendo los hombres quienes declararon una mayor ingesta ${ }^{26}$, la Oficina de Estudios y Políticas Agrarias en su reporte del año 2014, indica que, en la población chilena, el consumo per cápita de legumbres ha disminuido a una tasa media anual en la última década de $-2,3 \%$ (de 4,2 a 3,3 kg/habitante). Lo anterior podría deberse al cambio de conducta alimentaria de la población, a través de la sustitución de proteína vegetal por animal, producto de un mayor ingreso económico per cápita ${ }^{27}$. Resultados disimiles reportó una 


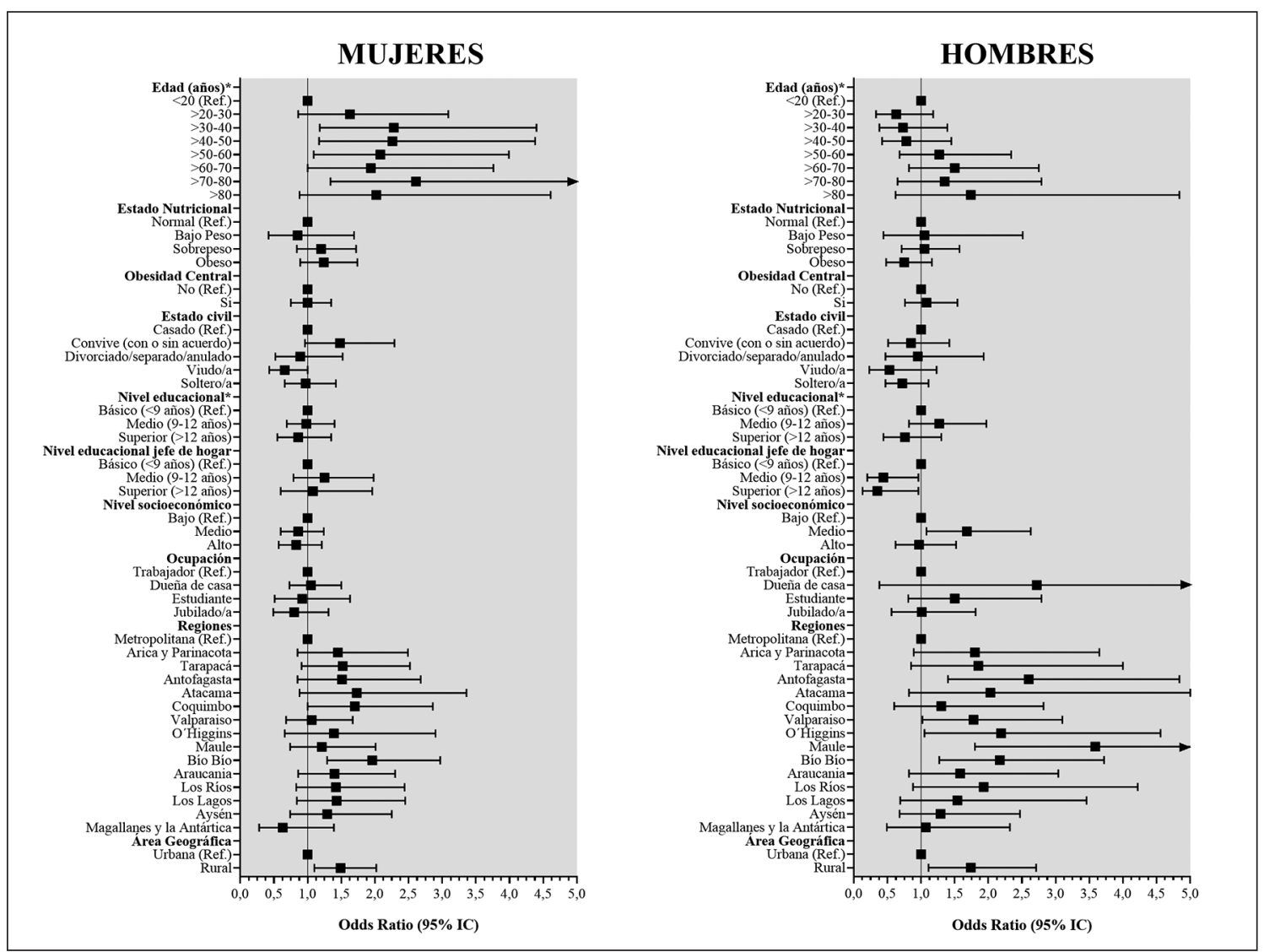

Figura 2. Probabilidad de cumplir la recomendación de ingesta de legumbres, según sexo y acorde a características sociodemográficas y antropométricas de la ENS 2016-2017, según modelo 2. Datos representados como Odds Ratio (OR) y su respectivo 95\% IC. Modelo 2 ajustado por variables sociodemográficas (edad, y educación). Las variables edad y nivel educacional no están ajustados por sí mismas. (Ref.): Referencia.

publicación canadiense del año 2017, que analizó los posibles ahorros anuales en costos de salud y sociales, del consumo de una alimentación alta en fibra dietética que incluya legumbres, señalando que al menos $13 \%$ de su población adulta consumía un promedio de $113 \mathrm{~g} /$ día, presentando los hombres un mayor consumo ${ }^{28}$.

En relación con las características de la población, tener una edad entre 60 y 80 años y ser mujer, aumentó significativamente la probabilidad de cumplir la recomendación, en los dos modelos. Resultados similares presentó la ENCA 2010, señalando que los grupos etarios entre 50-64 y $\geq 65$ años aumentaron la proporción de cumplimiento, a $25,2 \%$ y $29,0 \%$, respectivamente ${ }^{18}$.

Los participantes de la categoría jubilado/a presentaron mayor probabilidad de cumplimiento, solo para el modelo 1, respecto de personas laboralmente activas, lo que podría asociarse a lo antes descrito en el informe de ENCA 2010 en personas de $\geq 60$ años $^{18}$. Probablemente, para estos participantes el consumo de legumbres forma parte de sus hábitos alimentarios, además, podrían tener problemas dentales y al ser un alimento de consistencia blanda, sería más fácil su ingesta. Otra variable sería contar con tiempo para la elaboración de estos alimentos ${ }^{29}$. Se debe considerar, además, que población a partir de los 65 y/o 70 años recibe el beneficio del Programa Nacional de Alimentación Complementaria para el Adulto Mayor, que entrega un alimento (Crema años Dorados) a base de cereales y legumbres ${ }^{30}$. 
Este alimento contiene harinas de arvejas o lentejas extruidas (cerca de $37 \%)^{8}$, lo que podría aumentar la probabilidad de consumir legumbres en la dieta.

Los participantes con nivel de educación superior presentaron menores probabilidades de cumplir con la recomendación respecto de aquellos con nivel educacional básico, esto se podría explicar por la falta de tiempo para guisar legumbres, la publicidad y oferta de comida rápida. Sin embargo, esta asociación desapareció tras ajustar por variables sociodemográficas ${ }^{31}$. Contrariamente, un estudio realizado en 18 países reportó que las personas con nivel educacional superior, consumían más frutas, verduras y legumbres ${ }^{32}$. A diferencia de un estudio canadiense publicado el año 2012, que reportó que el nivel de educación no influyó en el consumo de legumbres, esto podría reflejar los diferentes antecedentes culturales de los canadienses, o la baja proporción de sus habitantes (16\%) con una educación inferior a la secundaria9 .

Los participantes que residían en las regiones del Maule y Biobío (zona centro sur), reportaron una mayor probabilidad de cumplir con la recomendación. Lo anterior podría estar relacionado con los hábitos alimentarios propios de la localidad. Al respecto, la ENCA 2010, señaló que 89,1\% de los habitantes de la macrozona centro-sur mostraron un consumo proporcional más alto que el resto del país ${ }^{18}$. Además, 77,7\% de los residentes de la zona metropolitana no cumplían con la recomendación de consumo de legumbres (16,4 g/ día), a diferencia de esta investigación en donde los habitantes que reportaron un menor consumo residían en la región de Magallanes y la Antártica chilena. Esto sugiere que las guías alimentarias implementadas por el MINSAL no han impactado en el consumo de legumbres de igual manera en todas las regiones del país ${ }^{17,18}$.

Los que residían en área rural, presentaron mayor probabilidad de cumplimiento, resultado que se mantuvo independiente del sexo. Esto podría estar relacionado con la producción local de estos alimentos y al bajo costo de ellos ${ }^{33}$. Resultados similares reportó la ENCA 2010, señalando un aumento de 4,1 g/día en habitantes de zonas rurales, respecto de urbanas $\left(17,7\right.$ a 21,8 g/día ${ }^{18}$.

Los solteros/as y los viudos/as, presentaron un menor cumplimiento de la recomendación. $\mathrm{Al}$ respecto, una publicación realizada en $\mathrm{Chile}^{8}$ señala que los adultos y los jóvenes de hoy, en su mayoría, no saben cocinar legumbres, o no las han visto preparar en casa ${ }^{8}$. En este contexto, el desarrollo de programas educativos que contemplen la preparación y degustación de legumbres para estimular su consumo, podría ser una estrategia para fomentar el consumo.

\section{Fortalezas y limitaciones}

Una de las fortalezas de este estudio, es la utilización de datos de la ENS 2016-2017, representativa de la población a nivel nacional. Como limitaciones, se identifica el auto-reporte de la ingesta de legumbres, lo que podría inducir a error en la estimación del consumo al identificarse solo la frecuencia de ingesta. Las variables de exposición también pueden estar sujetas a errores asociados al auto-reporte lo que puede influir en la asociación observada en el consumo de legumbres y estas variables. Finalmente, la naturaleza transversal del estudio no permite extraer ninguna inferencia causal de los resultados.

\section{Conclusión}

El consumo de legumbres a nivel nacional es bajo. Los participantes que tuvieron menor probabilidad de cumplir la recomendación fueron los viudos/as. Al contrario, los que presentaron una mayor probabilidad de cumplir la recomendación fueron las personas entre 60 y 80 años, los que residen en las regiones del Maule y Biobío y en área rural. A pesar de que los resultados difirieron al ser estratificados por sexo, se destaca que pertenecer a zonas rurales y a la región del Biobío se asoció con una mayor probabilidad de consumo en relación con sus contrapartes en ambos sexos.

Promover patrones dietéticos saludables como la ingesta de legumbres, a través de todo el ciclo vital, podría ser una estrategia alimentaria efectiva y de bajo costo para mantener una buena nutrición y salud. Los hallazgos de este estudio señalan la necesidad de fomentar el consumo de este grupo de alimentos.

Agradecimientos: Se agradece a todos los participantes de la ENS 2016-2017, al equipo profesional de la Escuela de Salud Pública, de la Facultad de Medicina de la PUC, quienes desarrollaron y aplicaron la ENS y al MINSAL. 


\section{Referencias}

1. FAO. Conclusiones del Año Internacional de las Legumbres 2017. Disponible: http://www.fao.org/zhc/ detail-events/es/c/470558/

2. FAO. Legumbres, Semillas Nutritivas Para Un Futuro Sostenible. 2016. Disponible: http://www.fao.org/3/ i5528s/i5528s.pdf

3. Durán E, Labraña AM. Calidad proteica de la dieta. In: Concepción EUd, editor. Indicadores de calidad de la dieta 2019; 71-89.

4. Mudryj AN, Yu N, Aukema HM. Nutritional and health benefits of pulses. Appl Physiol Nutr Metab. 2014; 39 (11): 1197-204.

5. Kouris-Blazos A, Belski R. Health benefits of legumes and pulses with a focus on Australian sweet lupins. Asia Pac J Clin Nutr. 2016; 25 (1): 1-17.

6. McCrory MA, Hamaker BR, Lovejoy JC, Eichelsdoerfer PE. Pulse consumption, satiety, and weight management. Adv Nutr. 2010; 1 (1): 17-30.

7. Asif M, Rooney LW, Ali R, Riaz MN. Application and opportunities of pulses in food system: a review. Crit Rev Food Sci Nutr. 2013; 53 (11): 1168-79.

8. Pinheiro A, Ivanovic C, Rodríguez L. Consumo de legumbres en Chile. Perspectivas y desafíos. Rev Chil Nutr 2018; 14-20.

9. Mudryj AN, Yu N, Hartman TJ, Mitchell DC, Lawrence FR, Aukema HM. Pulse consumption in Canadian adults influences nutrient intakes. Br J Nutr 2012; 108 Suppl 1: S27-36.

10. Marinangeli CPF, Curran J, Barr SI, Slavin J, Puri S, Swaminathan S, et al. Enhancing nutrition with pulses: defining a recommended serving size for adults. Nutr Rev. 2017; 75 (12): 990-1006.

11. Singh B, Singh JP, Shevkani K, Singh N, Kaur A. Bioactive constituents in pulses and their health benefits. J Food Sci Technol 2017; 54 (4): 858-70.

12. Rebello CJ, Greenway FL, Finley JW. A review of the nutritional value of legumes and their effects on obesity and its related co-morbidities. Obes Rev. 2014; 15 (5): 392-407.

13. Bouchenak M, Lamri-Senhadji M. Nutritional quality of legumes, and their role in cardiometabolic risk prevention: a review. J Med Food. 2013; 16 (3): 185-98.

14. Nouri F, Sarrafzadegan N, Mohammadifard N, Sadeghi $\mathrm{M}$, Mansourian M. Intake of legumes and the risk of cardiovascular disease: frailty modeling of a prospective cohort study in the Iranian middle-aged and older population. Eur J Clin Nutr 2016; 70 (2): 217-21.

15. Collaborators GD. Health effects of dietary risks in 195 countries, 1990-2017: a systematic analysis for the
Global Burden of Disease Study 2017. Lancet. 2019; 393 (10184): 1958-72.

16. Martínez-Sanguinetti MA, Leiva AM, Petermann-Rocha F, Villagrán M, Troncoso-Pantoja C, Celis-Morales C. Una de cada cinco muertes en el mundo se asocian a una alimentación no saludable: ¿Cuál es la realidad chilena? Rev Chil Nutr 2019; 46: 653-5.

17. MINSAL. Guías Alimentarias para la Población Chilena. Ministerio de Salud (MINSAL), Gobierno de Chile. 2013. Disponible: https://www.integra.cl/wp-content/ uploads/2017/01/GUIAS-ALIMENTARIAS.pdf

18. Amigo H, Bustos P, Pino P. Consumo según guías alimentarias. In: Universitaria E, editor. Alimentación y nutrición de los chilenos Encuesta Nacional de Consumo Alimentario 2018; 107-14.

19. MINSAL. Manual de aplicación de cuestionario F2, Encuesta Nacional de Salud 2016-2017. Ministerio de Salud (MINSAL), Gobierno de Chile. 2016. Disponible: http://epi.minsal.cl/encuesta-ens-descargable/

20. MINSAL. Encuesta Nacional de Salud 2016-2017. Ministerio de Salud (MINSAL), Gobierno de Chile. 2017. Disponible: https://www.minsal.cl/wp-content/ uploads/2017/11/ENS-2016-17_PRIMEROS-RESULTADOS.pdf

21. MINSAL. Presentación F1, cuestionario III Encuesta Nacional de Salud 2016-2017. Ministerio de Salud (MINSAL), Gobierno de Chile. 2016. Disponible: http:// epi.minsal.cl/encuesta-ens-descargable/

22. ESOMAR. The ESOMAR Standard Demographic Classification. European Society for Opinion and Marketing Research (ESOMAR). Hoffmeyer-Zlotnik J, Wolf C, editors. Advances in Cross-National Comparison Springer, Boston, MA.2003.

23. Petermann-Rocha F, Rocha C, Martinez-Sanguinetti MA, Leiva AM, Troncoso-Pantoja C, Villagran M, et al. [Association between adiposity and asthma]. Rev Med Chile 2019; 147 (6): 733-40.

24. WHO. Obesity: preventing and Managing the Global Epidemic. World Health Organization; 2000.

25. MINSAL. Orientación Técnica para la Atención de Salud de las Personas Adultas Mayores en Atención Primaria. Ministerio de Salud (MINSAL), Gobierno de Chile. 2016. Disponible: http://www.repositoriodigital.minsal.cl/ bitstream/handle/2015/440/Orientacion-tecnica-para-laatencion-en-salud-de-las-personas-adultas-mayores-enAP-MINSAL-Chile-2014-1.pdf?sequence=18isAllowed $=\mathrm{y}$

26. Vera V, Crovetto $M$, Valladares $M$, Oñate G, Fernández M, Espinoza V, et al. Consumo de frutas, verduras y legumbres en universitarios chilenos. Rev Chil Nutr 2019; 46: 436-22.

27. ODEPA. Evolución del Consumo Aparente de los 
Principales Alimentos en Chile: 2003-2013. Oficina de Estudios y Políticas Agrarias (ODEPA). 2014.

28. Abdullah MMH, Marinangeli CPF, Jones PJH, Carlberg JG. Canadian Potential Healthcare and Societal Cost Savings from Consumption of Pulses: A Cost-Of-Illness Analysis. Nutrients. 2017; 9 (7).

29. Hernández Galiot A, Pontes Torrado Y, Goñi Cambrodón I. Riesgo de malnutrición en una población mayor de 75 años no institucionalizada con autonomía funcional. Nutr Hosp 2015; 32: 1184-92.

30. MINSAL. Norma técnica de los programas alimentarios. 2016. Disponible: https://www.minsal.cl/ wp-content/uploads/2015/09/2016.04.20-Norma-T\%C3\%A9cnica-Prog.-Alimentarios-aprobada-por-Jur\%-
C3\%ADdica.pdf

31. Alarcón-Riveros M, Troncoso-Pantoja C, Amaya-Placencia A, Sotomayor-Castro M, Amaya-Placencia JP. Alimentación saludable en estudiantes de educación superior en el sur de Chile. Perspectivas en Nutrición Humana. 2019; 21: 41-52.

32. Miller V, Mente A, Dehghan M, Rangarajan S, Zhang $\mathrm{X}$, Swaminathan S, et al. Fruit, vegetable, and legume intake, and cardiovascular disease and deaths in 18 countries (PURE): a prospective cohort study. Lancet. 2017; 390 (10107): 2037-49.

33. Baginsky GC, Ramos CL. Situación de las legumbres en Chile: Una mirada agronómica. Rev Chil Nutr 2018; 45: 21-31. 